Original Article

\title{
The Correlation of Tumor Markers, Neutrophil/Lymphocyte Ratio and FDG PET/CT Measures in Pancreatic Cancer
}

\author{
Merve HAFIZOGLU ${ }^{1}$ (D) , Reyhan KOROGLU² (D) ,Hacer DEMIR $^{3}$ (D) , Erhan BOZKURT $^{1}$ (D) , \\ Emre HAFIZOGLU ${ }^{4}$ \\ ${ }^{1}$ Department of Internal Medicine, Afyonkarahisar Health Sciences University, Afyon, Turkey \\ ${ }^{2}$ Department of Nuclear Medicine, Afyonkarahisar Health Sciences University, Afyon, Turkey \\ ${ }^{3}$ Divsion of Medical Oncology, Department of Internal Medicine, Afyonkarahisar Health Sciences University, Afyon, Turkey \\ ${ }^{4}$ Clinic of Internal Medicine, Afyonkarahisar Public Hospital, Afyon, Turkey
}

\section{A B S T R A C T}

Background This review will outline the correlation of tumor markers and NLR (Neutrophil/ lymphocyte ratio) with measures such as MTV (mean total volume), TLG (total lesion glycolysis), and SUV (standard uptake value) in ${ }^{18} \mathrm{~F}$ FDG PET/CT in pancreatic carcinoma with the goal of selection of appropriate treatment modality and decrease rates of treatment failure and recurrences in pancreatic carcinoma by using tumor markers. In this way, we can predict the results of imaging modalities, using easy lab technics such as NLR and tumor markers.

Material and Methods 45 patients newly diagnosed with pancreatic cancer were included in the study. The diagnosis was confirmed by cytology. Their tumor marker levels (CA 19.9, CEA and AFP), NLR and PET/CT measurements (SUVmax, SUVmean, TLG, MTV) were obtained. Patients that were already diagnosed, followed up, or treated by the oncology department, were excluded.

Results When tumor markers were compared with PET/CT measurements (SUVmax, SUVmean, TLG, MTV and tumor size) there were no significant difference between them. Also, total uptake values of organs (liver, spleen, pancreas) were not related with tumor marker levels. However, there were positive significant correlation between tumor size and SUVmax, SUVmean, TLG ( $p=0.02, r: 0.347 ; p=0.022$, $\mathrm{r}: 0.340 ; \mathrm{p}=0.008, \mathrm{r}: 0.392$ ).

Conclusions Tumor markers may help diagnosing or managing of pancreatic malignities, but we cannot predict PET/CT results according to tumor marker levels. So, tumor markers must be used as an adjunctive method for diagnosing malignities. They cannot be major determiner for malignities. Diagnosing and following up malignities should be supported by other laboratory technics and imaging methods.

Keywords: CA 19.9, CEA, AFP, SUVmax, MTV, TLG.

\begin{tabular}{l|l} 
Address for Correspondence: \\
Merve Hafizoglu, MD
\end{tabular}




\section{Introduction}

Pancreatic cancer is an important health problem because of its aggressive behavior, it causes death in $95 \%$ of patients. Five-year overall survival (OS) rate after surgery is only about 10 to 20 percent whereas it is less than for metastatic pancreatic cancer. ${ }^{1,2}$

Cancer antigen 19.9 (CA 19.9) is a tumor marker present at high levels in some of malignities like bile duct, hepatocellular, colorectal, gastric, esophagus and pancreas cancer. It can be used to confirm the diagnosis of pancreas cancer, to evaluate the response to the treatment and the recurrence of pancreatic cancer. $^{3-5}$ Also, it can be elevated in benign conditions such as biliary tract obstruction, cholangitis, acute or chronic pancreatitis, liver cirrhosis, cystic fibrosis, thyroid diseases, inflammatory bowel disease or in normal, healthy population high levels can be detected. ${ }^{6}$ It's known that $5 \%$ of population is unable to synthesize CA 19.9 even if there are malign conditions. $^{7}$

Carcinoembryonic antigen (CEA) is a glycoprotein, and it has immunosuppressive and tumor cell adhesion properties, thus it facilitates metastasis and invasion of tumor cells..$^{8-13}$ In healthy individuals, CEA is present at very low levels in the blood because its production stops before birth. Although it is not tumor specific, its concentrations are raised in some types of cancer. ${ }^{14-18}$

Alfa-feto protein (AFP) is also produced during fetal development by the yolk sac and the liver. It plays a major role to screen liver cancer, preoperatively evaluation or postoperative monitoring and it can show advanced disease. Its levels can be raised not only in hepatocellular carcinoma, but also in other benign diseases and malignancies of testes and other germ cells. ${ }^{19-21}$

Neutrophil/lymphocyte ratio (NLR) is an indicator of systemic inflammation. We know that inflammation may play important roles in the development and progression of malignities. The presence of an elevated peripheral NLR has been recognized as a poor prognostic factor in various cancers.

$\mathrm{PET} / \mathrm{CT}$ (positron emission tomographycomputed tomography) is animaging method that combines functional imaging with anatomical images. The most common radiotracer on PET is $18 \mathrm{~F}$-flurodeoxyglucose (18F-FDG), which is a glucose analogue. Pancreatic malignities are usually associated with an overexpression of glucose transporter 1 and it causes increased 18F-FDG uptake on PET/CT. ${ }^{22} \mathrm{~A}$ main limitation of this imaging modality is the low spatial resolution and possibility of false positive uptake in normal structures or benign diseases, such as inflammatory processes. ${ }^{23}$

SUV max (maximum standardized uptake value) is a semi quantitative measure commonly used in 18F-FDG PET/CT. MTV (metabolic tumor volume), is a volumetric measurement of tumor cells with increased 18F-FDG uptake. The MTV on 18F-FDG PET/CT also demonstrates the metabolic activity, thereby it predicts the response to treatment and helps to determine the prognosis in head and neck cancers. In addition to SUVmax and MTV, TLG (total lesion glycolysis) constitutes another measurement derived from FDG $\mathrm{PET} / \mathrm{CT}$ that can be useful for predicting prognosis in some of malignities. ${ }^{24-27}$

This study will outline the correlation of tumor markers and NLR with measures such as MTV, TLG, and SUV in 18F FDG PET/CT in pancreatic carcinoma to select appropriate treatment modalities and decrease rates of treatment failure and recurrences in pancreatic carcinoma by using tumor markers. In this way, we can predict the results of imaging modalities, using easy laboratory technics such as NLR and tumor markers.

\section{Material and Methods}

This cross-sectional study was conducted between April 2016 and December 2018 in internal medicine outpatient clinics of the Afyonkarahisar Health Science University, Turkey. It was approved by the institutional review board of Afyonkarahisar Health Science University with the number 2019/145.

Forty-five patients newly diagnosed with pancreatic cancer were included in the study. The diagnosis was confirmed by cytology. Their tumor marker levels (CA 19.9, CEA and AFP), NLR and PET/CT measurements (SUVmax, SUVmean, TLG, MTV) were obtained. The interval between the FDG- 
Table 1. Mean values of tm markers and PET/CT measurements

\begin{tabular}{lllll} 
& Minimum & Maximum & Mean & Std Deviation \\
\hline Age & 36 & 84 & 62 & \\
Tumor size $(\mathrm{mm})$ & 19 & 97 & 41.6 & 10.1 \\
CA $19.9(\mathrm{U} / \mathrm{mL})$ & 0.60 & 10.000 & 1329.7 & 17.4 \\
CEA $(\mathrm{ng} / \mathrm{mL})$ & 0.99 & 71.29 & 10.2 & 2923.1 \\
AFP $(\mathrm{ng} / \mathrm{mL})$ & 0.86 & 9.48 & 2.5 & 14.2 \\
NLR & 0.76 & 27.72 & 5.56 & 1.4 \\
SUVmax & 3.0 & 17.2 & 8.1 & 6.55 \\
SUVmean & 2.0 & 10.7 & 4.5 & 3.5 \\
TLG & 14.8 & 1248 & 161.3 & 1.9 \\
MTV & 4.5 & 156 & 38.3 & 188.3 \\
\hline
\end{tabular}

NLR: neutrophil/lymphocyte ratio, TLG: total lesion glycolysis, MTV: metabolic tumor volum

$\mathrm{PET} / \mathrm{CT}$ evaluation and determining tumor markers was no more than one week. Tumor size was obtained by measure of greatest diameter of tumor with computed tomography. Patients that already diagnosed, followed up or treated by oncology department, were excluded. 18F-FDG-PET/CT scans were performed in accordance with a standard wholebody oncological protocol in each institution following the guidelines of the European Association of Nuclear Medicine.

\section{Statistical analysis}

The data collected were analyzed by SPSS for IBM, version 23.0 (SPSS, Turkey). Patient characteristics were reported using frequency and descriptive analyses. Tests of normality was determined by Shapiro-Wilk test. Correlation between variables was analyzed with Spearman's test. A p-value of less than 0.05 was considered to statistically significant.

\section{Results}

Overall, 45 patients (20 men and 25 women) were included in this study. The mean age of patients was $62 \pm 10.1$ year. The mean serum levels of tumor markers and NLR and PET/CT measurements are shown in Table 1.

Mean serum levels of CA 19.9 was $1329.7 \pm 2923.1 \mathrm{U} / \mathrm{mL}$. 17 of the patients had normal CA 19.9 levels (under $37 \mathrm{U} / \mathrm{Ll}$ ). 4 of them had levels above 10,000 U/mL.

Mean CEA levels were $10.2 \pm 14.2 \mathrm{ng} / \mathrm{mL}$ and nineteen patients had normal CEA levels (under $5 \mathrm{ng} / \mathrm{mL}$ ). However, AFP levels of all patients were at normal range (under $10 \mathrm{ng} / \mathrm{mL}$ ). Mean NLR were $5.56 \pm 6.55$. Three patients had very high levels of NLR (above 25) since they had bacterial co-infections.

When tumor markers were compared with PET/CT measurements there were no significant difference between them (Table 2). Also, total uptake values of organs (liver, spleen, pancreas) were not related with tumor marker levels.

Table 2. $\mathrm{P}$ values of correlations between tumor markers, NLR and PET-CT measurements

\begin{tabular}{lllllclll}
\hline & SUVmax & SUVmean & TLG & MTV & Tumor Size & Liver & Spleen & Pancreas \\
& & & & & & & & \\
\hline CA 19.9 & 0.503 & 0.464 & 0.961 & 0.845 & 0.660 & 0.751 & 0.439 & 0.811 \\
CEA & 0.322 & 0.228 & 0.487 & 0.379 & 0.499 & 0.643 & 0.319 & 0.071 \\
AFP & 0.854 & 0.884 & 0.152 & 0.166 & 0.095 & 0.105 & 0.498 & 0.708 \\
NLR & 0.315 & 0.186 & 0.468 & 0.478 & 0.912 & 0.512 & 0.252 & 0.696
\end{tabular}

SUVmax: maximum standardized uptake value, SUVmean: Mean standardized uptake value, TLG= total lesion glycolysis, MTV=metabolic tumor volume, $\mathrm{NLR}=$ neutrophil lymphocyte ratio $(\mathrm{P}<0.05$ significant $)$ 


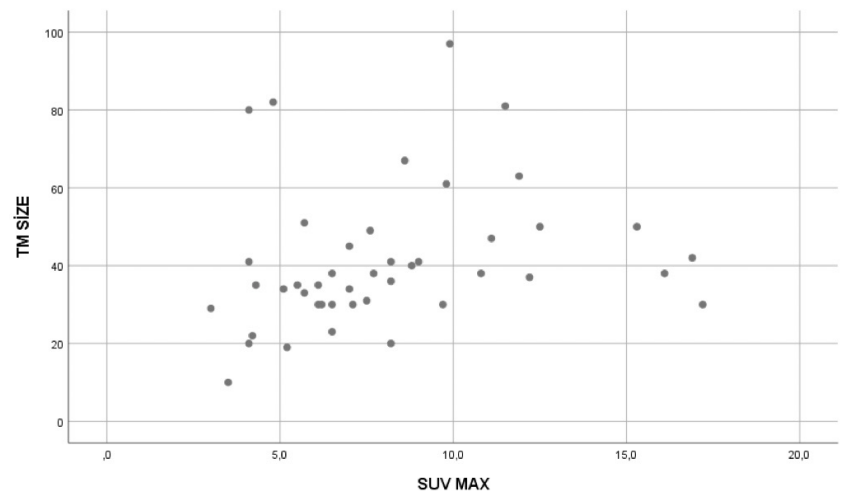

Graphic 1. Correlation between tumor size and SUVmax

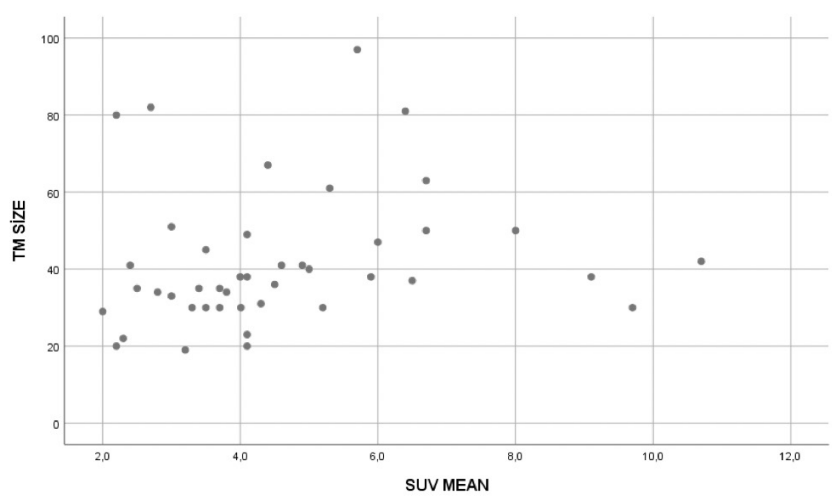

Graphic 2. Correlation between tumor size and SUVmean

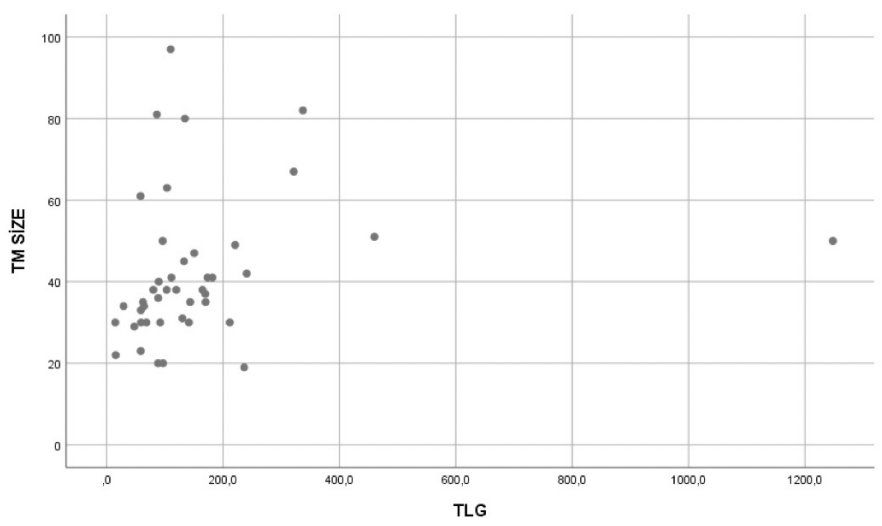

Graphic 3. Correlation between tumor size and TLG

Interestingly, we found that a negative correlation between CA 19.9 levels and SUVmax, SUVmean but it was not statistically significance ( $p=0.503, r:-0.102 ; p=0.464, r:-0.112)$. Likewise, between CEA levels and TLG, MTV there were negative insignificant correlation $(p=0.487$, r:-0.106; $p=0.379$, r:-0.134). Negative correlation continued between AFP and TLG, MTV also tumor size. Its level was also negligible $(p=0.152$, $\mathrm{r}:-0.217 ; \mathrm{p}=0.166, \mathrm{r}:-0.210 ; \mathrm{p}=0.095, \mathrm{r}:-0.252)$.
Between NLR and SUVmax, SUVmean there were negative insignificant correlation $(p=0.315$, $\mathrm{r}:-0.153 ; \mathrm{p}=0.186, \mathrm{r}:-0.201)$.

As expected, there were positive significant correlation between tumor size and SUVmax, SUVmean, TLG as shown in graphic 1,2 and 3. $(p=0.02, r: 0.347 ; p=0.022, r: 0.340 ; p=0.008$, $\mathrm{r}: 0.392)$.

\section{Discussion}

Tumor markers are used for detecting, diagnosing, managing certain types of cancer and also determining the progression of disease.

Carbohydrate antigen 19.9 also called cancer antigen 19.9 or sialylated Lewis antigen is a tumor marker that is used firstly in the management of pancreatic cancer. ${ }^{28}$ But it may be falsely positive in cases of biliary inflammation or obstruction. ${ }^{29}$ However, CA 19.9 may be undetectable in Lewis antigen-negative individuals even if they have advanced cancer. In our study 17 of patients have normal CA 19.9 value.

As in this study, CA 19.9 serum level of 100 $\mathrm{U} / \mathrm{mL}$ suggestive of unresectability or metastatic disease. $^{30}$ Our 4 patients that have distant metastasis, have up to $10,000 \mathrm{U} / \mathrm{mL}$ value of CA 19.9.

In pancreatic cancers, levels of CEA may reflect the tumor size, differentiation and metastasis. ${ }^{31}$ Also, its pancreatic juice levels can be used as a tumor marker. ${ }^{16,32-34}$ Preoperatively, high serum levels of CA 19.9 and CEA can be an indication of nonresectability or low chance of survival. While CA19.9 level is increased in both malignant and benign diseases, CEA increases only in malignant diseases. ${ }^{35}$ In our study mean value of CEA was $10.2 \pm 14.2 \mathrm{ng} / \mathrm{mL}$. Only 19 patients' results were under $5 \mathrm{ng} / \mathrm{mL}$, it can be explained by the presence of CEA-related glycoproteins. Unlike the literature, we did not find any correlation between CEA levels and tumor size.

AFP has a high specificity for a hepatocellular carcinoma. It can be used for screening hepatocellular carcinoma and its levels also can be elevated in liver cirrhosis and chronic hepatitis. ${ }^{36}$ We found that all AFP values were under $10 \mathrm{ng} / \mathrm{mL}$. According to these results, we can predict that in pancreas malignities even if 
there is distant metastasis (like liver metastasis), elevated AFP values are not always expected.

It is known that inflammation plays a very significant role in the development of cancer and may affect cancer patients' survival. ${ }^{37}$ Systemic inflammation supports tumor metastasis and progression. ${ }^{38}$ Because inflammatory cells and mediators generate a tumor related inflammatory microenvironment which plays vital roles in tumor progression and pathogenesis. ${ }^{39}$ The previous meta-analyses had showed that the prognostic value of preoperative NLR for patients with cancers, such as epithelial ovarian or upper urothelial tract, hepatocellular carcinoma and all solid tumors. ${ }^{40-43}$ In the same way, Li et al. ${ }^{44}$ indicated that high NLR was associated with poor overall survival, disease free survival, recurrence free survival in colorectal cancer. They had defined high NLR as values higher than $5 .{ }^{44,45}$ Also Fujii et al. ${ }^{46}$ found that high NLR were significantly associated with high SUVmax in the primary tumor of breast. They demonstrated that high NLR may be predictive of poor prognosis among patients with breast cancer 46 . In our study, the mean value of NLR was 5.56 \pm 6.55 . Contrarily, we did not detect any significant relation between NLR and SUVmax, SUVmean.

Sun et al. ${ }^{47}$ showed that SUVmax levels were associated with tumor size in pancreatic cancer patients. ${ }^{47}$ Like them, we also defined relation between tumor size and SUVmax, SUVmean, TLG.

As a result, tumor markers may help diagnosing or managing of pancreatic malignities but we can not predict PET/CT results according to tumor marker levels. So, tumor markers must be used as an adjunctive method for diagnosing malignities. They can not be major determiner for malignities. Diagnosing and following up malignities should be supported by other laboratory technics and imaging methods.

Unfortunately, our study has some limitations. First of all, it is a retrospective study and involves small sample size and all patients were selected from same hospital. Therefore, to say a relation between tumor markers and $\mathrm{PET} / \mathrm{CT}$ measurements, multi-center, large sample prospective studies are needed.

\section{Conflict of interest}

The authors declared that there are no potential conflicts of interest.

\section{Authors' Contribution}

Study Conception: $\mathrm{MH}$; Study Design: $\mathrm{MH}$; Supervision: EH, HD; Funding: EB; Materials: ABS, NU, FCH; Data Collection and/or Processing: RK; Statistical Analysis and/or Data Interpretation: RK, EB; Literature Review: $\mathrm{EH}$, $\mathrm{HD}$; Manuscript Preparation: $\mathrm{MH}$; and Critical Review: EH, HD.

\section{References}

1. Siegel RL, Miller KD, Jemal A. Cancer statistics. CA Cancer J Clin. 2015 Jan-Feb;65(1):5-29. doi:10.3322/caac. 21254

2. Karaman M. Effects of CA9 and TSPAN8 gene silencing in pancreatic cancer, an in vitro and in vivo study. Unpublished doctoral dissertation (supervisor: Yildirim H). Balikesir University, Institute of Science. Balikesir, 2018 (in Turkish).

3. Steinberg W. The clinical utility of the CA 19-9 tumor-associated antigen. Am J Gastroenterol. 1990 Apr;85(4):350-5.

4. Goonetilleke KS, Siriwardena AK. Systematic review of carbohydrate antigen (CA 19-9) as a biochemical marker in the diagnosis of pancreatic cancer. Eur J Surg Oncol. 2007 Apr;33(3):266-70. doi:10.1016/j.ejso.2006.10.004.

5. Boone BA, Steve J, Zenati MS, Hogg ME, Singhi AD, Barlett DL, Zureikat AH, Bahary N, Zeh HJ. Serum CA 19-9 response to neoadjuvant therapy is associated with outcome in pancreatic adenocarcinoma. Ann Surg Oncol. 2014 Dec;21:4351-8. doi: 10.1245/10434-014-3842-z.

6. Ito S, Gejyo F. Elevation of serum CA19-9 levels in benign diseases. Intern Med, 1999 Nov;38(11):840-1. doi:10.2169/internalmedicine.38.840.

7. Tempero MA, Uchida E, Takasaki H, Burnett AD, Steplewski Z, Pour PM. Relationship of carbohydrate antigen 19-9 and Lewis antigens in pancreatic cancer. Cancer Res. 1987 Oct;15;47(20):5501-3.

8. Gold P, Freedman SO. Specific carcinoembryonic antigen of human digestive system. J Exp Med. 1965 Sep;1;122(3):467-81.

9. Blumenthal RD, Hansen HJ, Goldenberg DM. Inhibition of adhesion, invasion, and metastasis by antibodies targeting CEACAM6 (NCA-90) and CEACAM5 (carcinoembryonic antigen). Cancer Res. 2005 Oct 1;65(19):8809-17.doi: 10.1158/0008-5472.CAN-05-0420.

10. Hostetter RB, Augustus LB, Mankarious R, Chi K, Fan D, Toth C, Thomas P, Jessup JM. Carcinoembryonic antigen as a selective enhancer of colorectal cancer metastasis. J Natl Cancer Inst. 1990 Mar 7;82(5):380-5.

11. Benchimol S, Fuks A, Jothy S, Beauchemin N, Shirota K, Stanners CP. Carcinoembryonic antigen, a human tumor marker, functions as an intercellular adhesion molecule. Cell. 1989 Apr 21;57(2):327-34. doi:10.1016/0092-8674(89)90970-7. 
12. Pignatelli M, Durbin H, Bodmer WF. Carcinoembryonic antigen functions as an accessory adhesion molecule mediating colon epithelial cell-collagen interactions. Proc Natl Acad Sci. 1990 Feb;87(4):1541-5. doi:10.1073/pnas.87.4.1541.

13. Medoff JR, Jegasothy BV, Roche JK. Carcinoembryonic antigen-induced release of a suppressor factor from normal human lymphocytes in vitro. Cancer Res. 1984 Dec; 44(12):5822-7.

14. Koness RJ. CEA: is it of value in colorectal cancer. R I Med. 1995 Jun78(6):164 6.

15. Tsavaris N, Vonorta K, Tsoutsos H, Kozatsani-Halividi D, Mylonakis N, Papagrigoriou D, Koutsiouba-Kazakou $\mathrm{P}$, Kosmidis P. Carcinoembryonic antigen (CEA), alphafetoprotein, CA 19-9 and CA 125 in advanced colorectal cancer (ACC). Int J Biol Markers. 1993 Apr-Jun;8(2):88-93.

16. Chevinsky AH. CEA in tumors of other than colorectal origin. Semin Surg Oncol. 1991;7(3):162-6. doi: 10.1002/ ssu.2980070309.

17. Gold P, Freedman SO. Demonstration of tumor-specific antigens in human colonic carcinomata by immunological tolerance and absorption techniques. J Exp Med. 1965 Mar;121(3): 439-62. doi: 10.1084/jem.121.3.439.

18. Garcia M, Seigner C, Bastid C, Choux R, Payan MJ, Reggio H. Carcinoembryonic antigen has a different molecular weight in normal colon and in cancer cells due to N-glycosylation differences. Cancer Res. 1991 Oct 15;51(20):5679-86.

19. Vastag B. Testicular cancer seen as 'stalking horse' for other cancers. J Natl Cancer Inst. 1999 Nov;91(21):1801-3.

20. Bei R, Mizejewski GJ. Alpha fetoprotein is more than a hepatocellular cancer biomarker: From spontaneous immune response in cancer patients to the development of an AFP based cancer vaccine. Curr Mol Med. 2011 Oct;11(7):564 81.

21. Sturgeon CM, Duffy MJ, Hofmann BR, Lamerz R, Fritsche HA, Gaarenstroom K, Bonfrer J, Ecke TH, Grossman HB, Hayes P, Hoffmann RT, Lerner SP, Löhe F, Louhimo J, Sawczuk I, Taketa K, Diamandis EP; National Academy of Clinical Biochemistry. National Academy of Clinical Biochemistry Laboratory Medicine Practice Guidelines for use of tumor markers in liver, bladder, cervical, and gastric cancers. Clin Chem. 2010 Jun;56(6):e1-48. doi: 10.1373/clinchem.2009.133124.

22. RReske SN, Grillenberger KG, Glatting G, Port M, Hildebrandt M, Gansauge F, Beger HG. Overexpression of glucose transporter 1 and increased FDG uptake in pancreatic carcinoma. J Nucl Med. 1997 Sep;38(9):1344-8.

23. Diederichs CG, Staib L, Vogel J. Values and limitations of 18F-fluorodeoxyglucose-positron-emission tomography with preoperative evaluation of patients with pancreatic masses. Pancreas. 2000 Mar;20(2):109-16. doi: 10.1097/00006676-200003000-00001.

24. Holgersson G, Ekman S, Reizenstein J, Bergqvist M, Pontén F, Uhlén $\mathrm{M}$, Magnusson $\mathrm{K}$, Jonnalagadda $\mathrm{P}$, Asplund A, Strömberg S, Linder A, Blomquist E, Liljeholm M, Lödén B, Hellström K, Bergström S. Molecular profiling using tissue microarrays as a tool to identify predictive biomarkers in laryngeal cancer treated with radiotherapy. Cancer Genomics Proteomics. 2010 Jan-Feb;7(1):1-7.
25. Schwartz DL, Harris J, Yao M, Rosenthal DI, Opanowski A, Levering A, Ang KK, Trotti AM, Garden AS, Jones CU, Harari P, Foote R, Holland J, Zhang Q, Le QT. Metabolic tumor volume as a prognostic imaging-based biomarker for head-and-neck cancer: Pilot results from Radiation Therapy Oncology Group protocol 0522. Int J Radiat Oncol Biol Phys. 2015 Mar 15;91(4):721-9. doi: 10.1016/j.ijrobp.2014.12.023.

26. Dibble EH, Alvarez AC, Truong MT, Mercier G, Cook EF, Subramaniam RM. 18F-FDG metabolic tumor volume and total glycolytic activity of oral cavity and oropharyngeal squamous cell cancer: Adding value to clinical staging. J Nucl Med. 2012;53:709-15. doi: 10.2967/ jnumed.111.099531.

27. Moon SH, Choi JY, Lee HJ, Son YI, Baek CH, Ahn YC. Prognostic value of 18F-FDG PET/CT in patients with squamous cell carcinoma of the tonsil: Comparisons of volume-based metabolic parameters. Head Neck. 2013 Jan;35(1):15-22. doi: 10.1002/hed.22904.

28. Shiozawa S, Tsuchiya A, Kim DH, Ogawa K. Prognostic significance of CA19 9 levels in patients with pancreatic cancer. Nihon Rinsho. 2006 Jan;64 Suppl 1:297-300 (in Japanese).

29. Marrelli D, Caruso S, Pedrazzani C, Neri A, Fernandes E, Marini M, Pinto E, Roviello F. CA199 serum levels in obstructive jaundice: clinical value in benign and malignant conditions. Am J Surg. 2009 Sep;198(3):333-339. doi: 10.1016/j.amjsurg.2008.12.031.

30. Ballehaninna UK, Chamberlain RS. The clinical utility of serum CA 19-9 in the diagnosis, prognosis and management of pancreatic adenocarcinoma: An evidence based appraisal. J Gastrointest Oncol. 2012 Jun;3(2): 10519. doi: 10.3978/j.issn.2078-6891.2011.021.

31. Zhang Q, Zeng L, Chen Y, Lian G, Qian C, Chen S, Li J, Huang K. Pancreatic Cancer Epidemiology, Detection, and Management. Gastroenterol Res Pract. 2016;2016:8962321. doi: 10.1155/2016/8962321.

32. Futakawa N, Kimura W, Yamagata S, Zhao B, Ilsoo H, Inoue T, Sata N, Kawaguchi Y, Kubota Y, Muto $\mathrm{T}$. Significance of K-ras mutation and CEA level in pancreatic juice in the diagnosis of pancreatic cancer. J Hepatobiliary Pancreat Surg. 2000;7(1):63-71. doi: 10.1007/s005340050156.

33. Basso D, Fabris C, Del Favero G, Angonese C, Meggiato $\mathrm{T}$, Infantino $\mathrm{A}$, Plebani $\mathrm{M}$, Piccoli A, Leandro $\mathrm{G}$, Burlina A, et al. Serum carcinoembryonic antigen in the differential diagnosis of pancreatic cancer: influence of tumour spread, liver impairment, and age. Dis Markers. 1988 Jul-Sep;6(3):203-7.

34. Sharma C, Eltawil KM, Renfrew PD, Walsh MJ, Molinari M. Advances in diagnosis, treatment and palliation of pancreatic carcinoma: 1990-2010. World J Gastroenterol. 2011 Feb 21;17(7):867-97. doi: 10.3748/wjg.v17.i7.867.

35. Juntermanns B, Radunz S, Heuer M, Hertel S, Reis H, Neuhaus JP, Vernadakis S, Trarbach T, Paul A, Kaiser MG. Tumor markers as a diagnostic key for hilar cholangiocarcinoma. Eur J Med Res. 2010 Aug;15(8):35761.

36. Stieber P, Heinemann V, Schalhorn A. Tumor markershow they should be applied. MMW Fortschr Med. 2005 May 19;147(20):35, 37-9 (in German).

37. Shalapour S, Karin M. Immunity, inflammation, and cancer: an eternal fight between good and evil. J Clin 
Invest. 2015 Sep;125(9):3347-55. doi: 10.1172/JCI80007.

38. McMillan DC. Systemic inflammation, nutritional status and survival in patients with cancer. Curr Opin Clin Nutr Metab Care. 2009 May;12(3):223-6. doi: 10.1097/MCO.0b013e32832a7902.

39. Mantovani A, Allavena P, Sica A, Balkwill F. Cancerrelated inflammation. Nature. 2008 Jul 24;454(7203):43644. doi: 10.1038/nature07205.

40. Yang Z, Gu JH, Guo CS, Li XH, Yang WC. Preoperative neutrophil-to-lymphocyte ratio is a predictor of survival of epithelial ovarian cancer: a systematic review and meta-analysis of observational studies. Oncotarget. 2017 Jul 11;8(28):46414-24. doi: 10.18632/oncotarget.16793.

41. Vartolomei MD, Kimura S, Ferro M, Vartolomei L, Foerster B, Abufaraj M, Shariat SF. Is neutrophilto-lymphocytes ratio a clinical relevant preoperative biomarker in upper tract urothelial carcinoma? A meta-analysis of 4385 patients. World J Urol. 2018 Jul;36(7):1019-29. doi: 10.1007/s00345-018-2235-5.

42. Sun XD, Shi XJ, Chen YG, Wang CL, Ma Q, Lv GY. Elevated preoperative neutrophil lymphocyte ratio is associated with poor prognosis in hepatocellularcarcinoma patients treated with liver transplantation: a metaanalysis. Gastroenterol Res Pract. 2016;2016:4743808. doi: 10.1155/2016/4743808.

43. Paramanathan A, Saxena A, Morris DL. A systematic review and metaanalysis on the impact of pre-operative neutrophil lymphocyte ratio on long term outcomes after curative intent resection of solid tumours. Surg Oncol. 2014 Mar;23(1):31-9. doi: 10.1016/j.suronc.2013.12.001.

44. Li H, Song J, Cao M, Wang G, Li L, Zhang B, Li, Y, $\mathrm{Xu} \mathrm{W}$, Zheng J. Preoperative neutrophil-to-lymphocyte ratio is a more valuable prognostic factor than plateletto-lymphocyte ratio for nonmetastatic rectal cancer. Int Immunopharmacol. 2016 Nov;40:327-331. doi: 10.1016/j. intimp.2016.09.014.

45. Ishizuka M, Nagata H, Takagi K, Iwasaki Y, Shibuya N, Kubota K. Clinical significance of the C-reactive protein to albumin ratio for survival after surgery for colorectal cancer. Ann Surg Oncol. 2016 Mar;23(3):900-7. doi: 10.1245/s10434-015-4948-7.

46. Fujii T, Yanai K, Tokuda S, Nakazawa Y, Kurozumi S, Obayashi S, Yajima R, Hiraka T, Shirabe K. Relationship Between FDG Uptake and Neutrophil/Lymphocyte Ratio in Patients with Invasive Ductal Breast Cancer. Anticancer Res. 2018 Aug;38(8):4927-31. doi: 10.21873/ anticanres.12809

47. Sun Y, Duan Q, Wang S, Zeng Y, Wu R. Diagnosis of pancreatic cancer using 18F-FDG PET/CT and CA19-9 with SUVmax association to clinical characteristics. J BUON. 2015 Mar-Apr;20(2):452-9. 